\title{
Polypharmacy in otolaryngologic secretory senescence
}

\author{
Elio Maria Cunsolo ${ }^{1}$, Pasquale Viola² \\ ${ }^{1}$ ENT Clinic, Modena, Italy; ${ }^{2}$ UO Audiology, Department of Experimental and Clinical Medicine, \\ "Magna Graecia" University, Catanzaro, Italy
}

The progressive aging of the population is a widely documented figure worldwide. Just as most individuals can lead longer lives, it is equally true that most of them experience a period of their life, specifically that of older age, characterized by the coexistence of multiple diseases. The growing number of elderly people with multimorbidity entails the need of treatment regimens that require the simultaneous administration of multiple drugs, in complex therapy regimens. This represents a real medical challenge, intended to an ever-increasing impact in the years to come. "Polypharmacy", generally understood as the simultaneous use of multiple drugs in the same patient, is obviously the rational response to the treatment of complex pathological pictures in aged individuals. Conversely, this polypharmacy implies a growing concern regarding the inappropriate and not strictly necessary simultaneous intake of a large number of drugs. This situation can lead to an actual change in the benefits of each single drug towards adverse effects, when it is taken in combination with other drugs, in complex and prolonged therapeutic regimens. This is precisely the "heart" of the clinical problem of polypharmacy and the adverse effects it causes. These considerations are particularly felt in the Ear-Nose-Throat (ENT) field, in which the specialist, generally lacking an adequate doctrinal and clinical background in gerontology and geriatrics, is called to evaluate and treat complex pathological pictures that embrace and overlap in conditions of multimorbidity, in regime of polypharmacy. The following displayed order will be adopted to discuss this complex issue: current definitions of polypharmacy, current concepts of clinical pharmacology of the elderly, polypharmacy in ENT clinical practice and in the secretory senescence of head-neck structures.

Key words: polypharmacy, pharmacokinetic, elderly

\section{ACTUAL DEFINITIONS OF "POLYPHARMACY"}

In a recent systematic review about the concept of polypharmacy, Masnoon et al. ${ }^{1}$ have found 138 definitions of this term. The $80.4 \%$ of these evaluate just the daily number of drugs, the $10.9 \%$ associate it with the duration of therapy and only the $6.4 \%$ consider the degree of appropriateness of the poly-pharmacological treatment implemented. In the definitions based exclusively on the numerical criterion, there is a wide variability in the range of this parameter and of the qualitative terms associated with it, such as "minor", "moderate", "major", "excessive" or "severe". The most commonly used criterion is the one that considers "Polypharmacy" as the intake of at
This is an open access article distributed in accordance with the CC-BY-NC-ND (Creative Commons Attribution-NonCommercial-NoDerivatives 4.0 International) license. The article can be used by giving appropriate credit and mentioning the license, but only for non-commercial purposes and only in the original version. For further information: https://creativecom-

mons.org/licenses/by-nc-nd/4.0/deed.en

(c) Copyright by Società Italiana di Gerontologia e Geriatria (SIGG) 
least 5 drugs per day, statistically followed by a more restrictive criterion, which raises this value to least 6 drugs per day. Two systems can be identified among the ones that combine the daily number of drugs taken with the temporal number of the duration of their usage. The first ("long time use") considers "Polypharmacy " taking only 2 drugs per day, if this has occurred for at least 240 days. Conversely, with regimens that consider the intake of at least 5 drugs per day, the "Polypharmacy" occurs after at least 90 continuous days of use. Studies that deal with the degree of "appropriateness" of the polypharmacy implemented regime are a minority, representing only $6.4 \%$. Polypharmacy is considered adequate when it leads to the optimisation of medications for patients with complex and/or multiple conditions, where medicine usage agrees with best evidence, while it is defined inappropriate in case of duplication of medications, drugdrug interactions, medications used to treat the side effects of other medications, and medications which are unnecessary for a specific patient.

Wastensson et al. ${ }^{2}$ have created a graphic framekork relating to the clinical consequences of polypharmacy in the elderly (Fig. 1).

Among the 110 studies taken into consideration by Masnoon et al. ${ }^{1}$, only 4 (3.6\%) use coded parameters to evaluate the appropriateness of the polypharmacy, such as the "Beers Criteria". The latter are edited by the American Geriatrics Society (AGS) for Potentially Inappropriate Medication (PIM) Use in Older Adults ${ }^{3}$, of which a very recent update is available.

A wide use of these criteria is also recommended by the ENT specialist.

In conclusion, at present it can be said that it does not exist any standard definition of what polypharmacy constitutes. In general, polypharmacy can be defined as a count of drugs and had to include some kind of clinical judgments about the quality/appropriateness of polypharmacy. When polypharmacy is defined as a simple count of the number of drugs, a cut-off of five or more concurrently is most frequently used.

\section{CURRENT CONCEPTS OF CLINICAL PHARMACOLOGY FOR THE ELDERLY}

The knowledge of the clinical pharmacology of the elderly patient needs the clarification of the main aspects that characterize it ${ }^{4}$. These include: age-related changes in pharmacokinetics and pharmacodynamics, drug interactions (drug to drug interactions) and, at last, drug-disease interactions (drug to disease interactions).

\section{Alterations of the pharmacokinetic}

These affect all the phases of the pharmacokinetics of

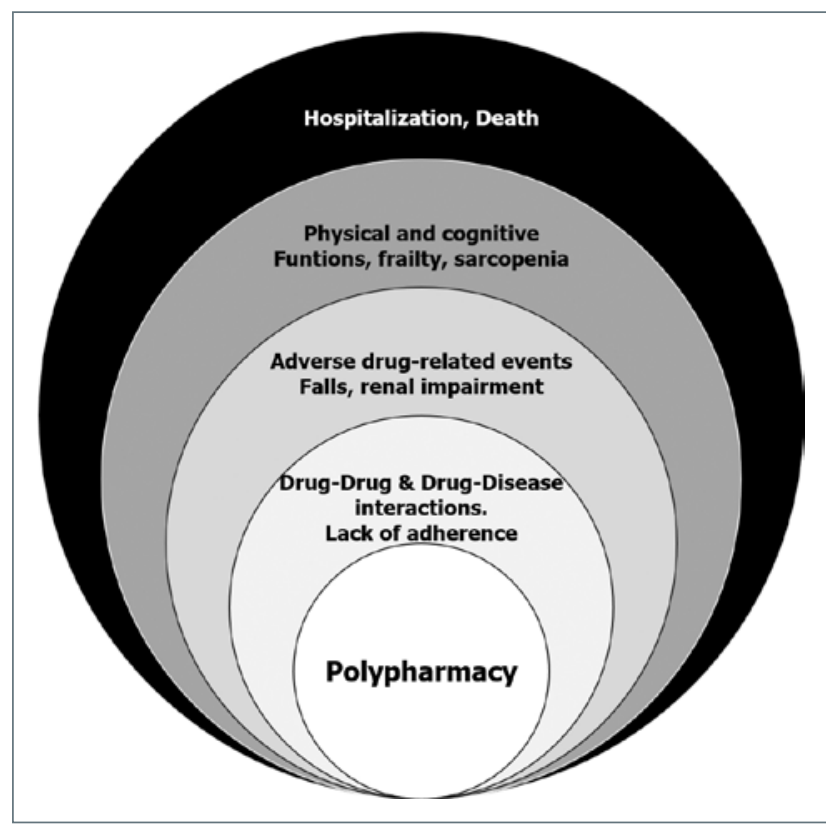

Figure 1. Framework for polypharmacy and conceptual classification of outcomes (from Wastesson et al., 2018, mod.) ${ }^{2}$.

drugs: absorption, distribution, metabolism, and elimination. The presence and severity of any frail condition is an important risk factor for any adverse drug reactions related to changes in pharmacokinetics ${ }^{5}$. The pharmacokinetic alterations of absorption concern the intake of oral medications. Indeed, with advancing age, numerous changes in the physiology of the gastro-intestinal tract occur. Variations in $\mathrm{pH}$ may be due to the establishment of atrophic gastritis and/or to the ever-increasing use of pump inhibitors drugs. Gastric emptying is delayed, as well as both the intestinal motility and the number of intestinal cells functionally efficient for absorption are reduced. Intestinal blood flow is cut down by about 30\% compared to young age. It is also important the decrease in the first-pass hepatic extraction, that occurs with aging, resulting into a systemic bioavailability enhancement for drugs such as propranolol and labetalol, and a reduced bioavailability of some prodrugs such as enalapril and codeine, after oral administration ${ }^{6}$. Distribution is another element of pharmacokinetics that significantly changes with age. Body fat, as a proportion of body weight, rises from 18 to $36 \%$ in men, and from 33 to $45 \%$ in women from age 20 to 70 years, whereas lean body mass decreases by $19 \%$ in men and by $12 \%$ in women and plasma volume decreases by $8 \%$ from age 20 to 80 years. Total body water decreases by $17 \%$ from age 20 to 80 years, and extracellular fluid volume decreases by $40 \%$ from 20 to 65 years of age. In addition, cardiac output declines of approximately 1\%/year from age 30 years, and brain and cardiac vessel blood flow rates decline $0.35 \%$ to 
$0.5 \% /$ year, respectively, beyond age 25 years. In addition, frailty and concurrent diseases may result in substantial changes in the serum concentrations of the two major drug-binding plasma proteins: albumin, which binds acidic drugs, decreases, whereas $\alpha 1$-acid glycoprotein, which binds basic drugs, remains the same or rises. As a result of these factors, the volume of distribution of water-soluble (hydrophilic) drugs is generally decreased and that of fat-soluble (lipophilic) drugs is increased. Moreover, changes in volume of distribution can directly affect the loading doses of medications. Decreases in serum albumin concentration can lead to a reduction in the degree of plasma protein binding of acidic drugs, such as naproxen, phenytoin, tolbutamide, and warfarin, therefore increasing the unbound fraction of the drug. Increases in $\alpha 1$-acid glycoprotein because of inflammatory disease, burns, or cancer can lead to enhancement in the degree of plasma protein binding of basic drugs such as lidocaine, $\beta$-blockers, quinidine, and tricyclic antidepressants, thus reducing the unbound fraction of the drug. Plasma protein binding changes can alter the relationship of unbound (free) and total (unbound plus bound) plasma drug concentrations, making drug concentration interpretation more difficult. In these cases, the measurement of free plasma drug concentrations may be preferable to the usual dosage of total plasma drug concentrations ${ }^{7}$. Permeability across the blood-brain barrier may also be altered in older adults, affecting distribution of drugs into the central nervous system (CNS). Cerebrovascular $\mathrm{P}$-glycoprotein is responsible for the transport of drugs across the blood-brain barrier. As a result, the brain of older adults may be exposed to higher levels of drugs ${ }^{8}$. Although drug metabolism can occur in numerous organs, most of the available data concern the effects of aging on the liver. Variations in drug metabolism and those resulting in altered drug clearance are a major source of variability in the response to medications in older adults. Hepatic metabolism of drugs depends on perfusion, liver size, activity of drug-metabolizing enzymes, transporter activity, and protein binding, all of which may be altered by aging ${ }^{9}$. Age-associated reductions in hepatic blood flow can reduce the clearance of high hepatic extraction ratio drugs such as amitriptyline, lidocaine, morphine, diltiazem, and propranolol.

Renal excretion is a primary route for the elimination of many drugs and their metabolites. Aging is associated with a significant reduction in renal mass, and number and size of nephrons. In addition, the glomerular filtration rate (GFR), tubular secretion, and renal blood flow decrease approximately of $0.5,0.7$, and $1 \% /$ year, respectively, in those older than 20 years. At all ages, these three parameters are lower in women than in men ${ }^{10}$. However, older adults are a heterogeneous group, with up to one third of healthy older adults having no decrement in renal function as measured by creatinine clearance, a surrogate for glomerular filtration. In addition, tubular secretion and glomerular filtration may not decline in parallel. Changes in kidney function with aging may be associated with hypertension or heart disease rather than with aging itself. The estimation of creatinine clearance $(\mathrm{CrCl})$ serves as a useful screen for renal impairment instead of the use of serum creatinine (SCr), which is an imperfect marker of renal function in older adults because of the reduction of muscle mass with advancing age (i.e., a normal serum creatinine level does not match with normal renal function in older adults).

The most commonly used estimation equation for creatinine clearance used for dosage adjustment in older adults is the Cockcroft and Gault equation:

$$
\text { creatinine clearance }=\frac{(140-\text { age }) \times(\text { actual body weight })}{72 \times S C r}
$$

where age is in years, actual body weight in kilograms, and serum creatinine concentration in milligrams per deciliter. For females, multiply the result by $0.85{ }^{11}$.

The risk of adverse clinical consequences is likely increased for drugs with a narrow therapeutic index (e.g., digoxin, aminoglycosides, chemotherapeutics). Consensus guidelines for oral dosing of primarily renally cleared drugs in older adults have been developed ${ }^{12}$. Medications to avoid in older adults with $\mathrm{CrCl}$ lower than $30 \mathrm{~mL} / \mathrm{min}$ include chlorpropamide, colchicine, cotrimoxazole, glyburide, meperidine, nitrofurantoin, probenecid, spironolactone, and triamterene. Oral medications with recommended dosage adjustments for reduced renal function in older adults include acyclovir, amantadine, ciprofloxacin, gabapentin, memantine, metformin, ranitidine, rimantadine, and valacyclovir. Dosage adjustment for renal impairment is easily accomplished once $\mathrm{CrCl}$ has been estimated using information provided in the package insert or other drugdosing reference sources.

\section{Altered pharmacodynamics}

Most studies documenting age-related differences in pharmacodynamics have focused on medications acting on the CNS and cardiovascular system. Theoretically, altered pharmacodynamics could be due to two basic mechanisms: a) altered sensitivity, because of changes in receptor number or affinity or changes in postreceptor response; b) age-related impairment of physiologic and homeostatic mechanisms.

There are a lot of drugs for which there is reasonable documentation of altered drug sensitivity in older adults. There is evidence that older adults are less responsive to $\beta$-blockers and $\beta$-agonists ${ }^{13}$. There is 
also good evidence that older adults are more sensitive to the effects of benzodiazepines. Using psychomotor testing, this has been established for diazepam, flurazepam, loprazolam, midazolam, nitrazepam, and triazolam ${ }^{14}$. Enhanced sensitivity has also been demonstrated for opioids, metoclopramide, dopamine agonists, levodopa, and antipsychotics. Age-related changes in pharmacodynamics have been reported for calcium channel blockers (increased hypotensive and bradycardic effects) and warfarin (increased risk of bleeding), but not with angiotensin-converting enzyme inhibitors or angiotensin receptor blockers ${ }^{15}$.

Physiologic and homeostatic changes in older adults may affect drug responses, altering baseline performance and the ability to compensate the effects of medications. Examples of homeostatic mechanisms that may become impaired with advanced age include postural or gait stability, orthostatic blood pressure responses, thermoregulation, cognitive reserve, bowel and bladder function ${ }^{16}$. The loss of efficiency of homeostatic mechanisms puts older adults at risk of symptomatic orthostasis and falls (antihypertensives, antipsychotics, and tricyclic antidepressants), urinary retention and constipation (drugs with anticholinergic properties), falls and delirium (with virtually every sedating drug) and accidental hypothermia or heat stroke (neuroleptics). Medications are a common contributor to geriatric syndromes such as falls, delirium, functional decline and constipation ${ }^{17}$.

\section{DRUG-DRUG INTERACTIONS}

Drug-drug interactions can be defined as the resulting effect or consequence that one drug has on another when co-administered ${ }^{18}$. The two major types of drugdrug interactions include pharmacokinetic interactions, wherein drug absorption, distribution, metabolism, and excretion are affected, and pharmacodynamic interactions, wherein pharmacologic effects are altered. Drugs may also interact with food, nutritional status, herbal products, alcohol, and preexisting disease ${ }^{19,20}$. Drug interactions involving drug distribution are primarily related to altered plasma protein binding. Although a number of drugs may displace other drugs from plasma protein-binding sites, especially acids such as salicylate, valproic acid and phenytoin, this type of drug interaction is rarely clinically significant. Inhibition of renal clearance of one drug by another drug can also result in clinically significant effects ${ }^{21}$. Many of these drug-drug interactions involve competitive inhibition of tubular secretion of anionic or cationic drugs. Cationic agents include amiodarone, cimetidine, digoxin, procainamide, quinidine, ranitidine, trimethoprim and verapamil. Anionic agents include cephalosporins, indomethacin, methotrexate, penicillins, probenecid, salicylates and thiazides.

Drug interactions with herbal and over-the-counter
(OTC) products are frequently overlooked. In one series, $52 \%$ of all moderate- or high-risk interactions occurred between prescription drugs and herbal and/ or OTC products. The interaction potential of herbal products is enhanced because of frequent contamination with heavy metals and adulteration with prescription drugs (e.g., nonsteroidal anti-inflammatory drugs [NSAIDs], corticosteroids, psychotherapeutics, and phosphodiesterase-5 inhibitors, such as sildenafil) ${ }^{22}$. Some drugs may alter the response of another drug and produce adverse effects (pharmacodynamic interactions). A good example of this is the synergistic effect of taking more than one anticholinergic agent concurrently, which can result in delirium, urinary retention, constipation, and other problems. Other examples include additive bradycardia when $\beta$-blockers are administered concurrently with verapamil or diltiazem, additive hypotension when several antihypertensives are administered concurrently and sedation or falls when several CNS depressants (e.g., benzodiazepines, sedative-hypnotics, antidepressants, neuroleptics) are administered concurrently.

Drug interactions can also be considered in a broader sense when they involve medications that can affect and can be affected by disease states (drug-disease interactions). Older adults are at higher risk for adverse outcomes with drug-disease state interactions because of alterations in homeostatic mechanisms, decreased physiologic reserve, and multiple comorbidities. Avoiding inappropriate medications, and identifying medication-related adverse events and drug interactions, coupled with patient participation, can have favorable effects on patient outcomes ${ }^{23}$. Expert panels in United States have developed guidelines to identify potentially clinically important Drug-Disease State interactions ${ }^{3}$. Implicit criteria, such as the Screening Tool of Older Person's Prescriptions (STOPP) and the Screening Tool to Alert doctors to Right Treatment (START), may be more advantageous when applying patient specific characteristics ${ }^{24-26}$.

In conclusion: polypharmacy increases the risk of adverse drug reactions and hospitalizations in geriatric patients. Rational deprescribing in the elderly of anticholinergics, benzodiazepines, antipsychotics, opioids, and proton pump inhibitors in selected patients may be a good first step to reducing this risk ${ }^{27}$.

\section{POLYPHARMACY IN OLDER PATIENTS WITH OTOLARYNGOLOGIC DISEASES}

In older people with otolaryngologic complaints, the impact of polypharmacy/overmedication causes significant problems in the following clinical conditions: 
balance disorders, xerostomia, sialorrhea, dysphagia, laryngo-pharyngeal reflux, sinusitis, and post-nasal drip ${ }^{28}$. The most common impact of overmedication in older adults is the impact on balance, predisposing affected patients to falls.

In 2010, the American Geriatric Society and the British Geriatric Society published a Clinical Practice on Prevention of falls - AGS/BGS-CPG recommendations ${ }^{29}$. These guidelines identify a set of Multifactorial or Multicomponent Interventions to Address Identified Risk(s) and Prevent Falls. Among these interventions the Minimization of Medications is of primary importance. Medications have consistently been associated with risk of falls. The strongest risk associations occur with psychotropic medications and polypharmacy. The strongest evidence supports withdrawal of psychotropic medication, as a single intervention and as a component of multifactorial and multicomponent intervention. If discontinuation of a particular high-risk medication is not possible because of medical conditions, dose reduction should be considered. Although some clinicians believe that selective serotonin reuptake inhibitors (SSRIs) are generally safer to use in older adults than tricyclic antidepressants, in terms of fall prevention, evidence is building that SSRIs increase fall risk as much as the older tricyclic antidepressants. Reduction of psychotropic medication as a single intervention has been found to reduce fall rate, whereas assessment, adjustment, and discontinuation of medication regimens as part of a multifactorial intervention have also been found to be effective in reducing falls.

\section{Polypharmacy in Older PatientS and Secretory SENESCENCE}

The most important clinical aspects in this area essentially concern the rhino-sinusal district and, above all, that set of anatomical and functional structures involved in swallowing. As with all systems in the body, the nose and paranasal sinuses change with aging. Presbynasalis refers to the constellation of gross and cell-level changes that occur in the aging sinonasal tract ${ }^{30}$. The immune system (mucosal and systemic) becomes less responsive with age, a process known as immunosenescence. The incidence of rhinorrhea increases as the population ages. Though nasal volumes increase with age, other age-related changes may result in the perception of increasing nasal obstruction with age. A recent publication ${ }^{31}$ examined the prevalence of clear anterior rhinorrhea in the population of Pittsburgh by means of cross-sectional survey. Rodriguez \& coll. discovered that $72 \%$ of older participants (average age 85 years) reported daily nasal drip and only $8 \%$ of younger participants (average age 20 years) reported the same. The older patients were also more bothered by nasal drip and would be more likely to seek treatment for the issue, particularly those patients with more frequent dripping. The effects of the autonomic nervous system are also involved. The influence of the sympathetic nervous system increases with age, but there is decreased sensitivity of the alpha- and betareceptors and the overall net effect may be a decrease in sympathetic tone, as compared to the more constant parasympathetic tone. This would be expected to lead to an increase in glandular activity ${ }^{32}$. There is significant alteration in the mechanics of the aging nose, resulting in perturbation of airflow. These effects, again, appear to be multifactorial, with no single change explaining all of the effects. Disruption in expected nasal cycle variation would be another age-related change contributing to the feeling of congestion in the elderly, possibly due to the increased risk of drying ${ }^{33}$. There are no published studies correlating nasal and sinus complaints with medication usage, hence this paragraph should be interpreted as "expert opinion." All otolaryngologists encounter patients on a daily basis with complaints of sinus or postnasal drip and these may be caused by medications. Anticholinergic and diuretic medications impair mucociliary function by reducing the sol layer of the mucus blanket. Reductions in this layer lead to ciliary dysfunction and mucus stasis. When this mucus reaches the posterior choana, it may adhere to the pharyngeal mucosa and be perceived as postnasal drip. A common observation by otolaryngologists is that patients who complain of postnasal drip typically have dry, adherent mucus rather than excessive amounts of mucus. Topical anticholinergic medications (ipratropium) are effective in reducing the copious amounts of clear nasal drainage in vasomotor rhinitis, often referred to as "senile drip", but do not reduce symptoms for the patient with common sensation of postnasal drip. Moreover, treatment with antihistamines and nasal steroid sprays are often not beneficial, may even exacerbate symptoms and lead to bleeding because of the drying effect on nasal mucosa. It is worthwhile periodically stopping these medications as an empiric trial.

\section{PolyphaRmacy AND SALIVARY SECRETORY DISORDERS}

The effect of medications on swallowing function is often underappreciated. Otolaryngologists who evaluate patients with swallowing disorders often encounter patients with swallowing difficulties caused by diuretics, anticholinergics, or centrally acting sedatives. Because swallowing functional reserve decreases with increasing age, even minimal impairment of lubrication or motor coordination can tip relatively normal swallowing function into dysphagia. Xerostomia is a frequently encountered sequelae of taking medications with anticholinergic effects. The main clinical problem of polypharmacy 
in secretory senescence in Otolaryngology is, in fact, represented by the role of drugs in causing disorders of salivary secretion. "Too many medications - not enough saliva": it is the significant title of a recent paper by David Ebling, which effectively summarizes the enormous clinical significance of this problem ${ }^{34}$. In recent years, complete rewiews have been published on the subject, which are also easily available and on the web. Salivary Secretory Disorders, Inducing Drugs, and Clinical Management ${ }^{35}$ is a review of 2015, available at: https://www.ncbi.nlm.nih.gov/pmc/articles/ PMC4615242/pdf/ijmsv12p0811.pdf.

Very important are the three rewiews sponsored by the World Workshop on Oral Medicine VI:

1 World Workshop on Oral Medicine VI: a systematic review of medication-induced salivary gland dysfunction: prevalence, diagnosis, and treatment ${ }^{36}$;

2 World Workshop on Oral Medicine Vl: a systematic review of medication-induced salivary gland dysfunction ${ }^{37}$;

3 a guide to medications inducing salivary gland dysfunction, xerostomia, and subjective sialorrhea: a systematic review sponsored by the World Workshop on Oral Medicine VI ${ }^{38}$ (https://link.springer.com/content/pdf/10.1007/s40268-016-0153-9.pdf).

\section{CONCLUSIONS}

The polypharmacy problem has a significant impact in the clinical management of the geriatric patient with polymorbidity. It includes important aspects of daily clinical practice in ENT, with important implications in the field of secretory senescence. This topic involves multi-disciplinary knowledge and is worthy of a wider monografic paper.

\section{References}

1 Masnoon N, Shakib S, Kalisch-Ellett L, et al. What is polypharmacy? A systematic review of definitions. BMC Geriatr 2017;17:230 https://doi.org/10.1186/s12877-017-0621

2 Wastesson JW, Morin L, Tan ECK, et al. An update on the clinical consequences of polypharmacy in older adults: a narrative review. Exp Op Drug Saf 2018;17-2:1185-96. https://doi.org/10.1080/14740338.2018.1546841

3 Fick DM, Semla TP, Steinman M, et al. American Geriatrics Society 2019 updated AGS Beers Criteria ${ }^{\circledR}$ for potentially inappropriate medication use in older adults. J Am Geriatr Soc 2019;67:674-94. https://doi.org/10.1111/ jgs. 15767

4 Slattum PW, Ogbonna KC, Peron EP. The pharmacology of aging. In: Fillit HM, Rockwood K, Young J, Eds. Brocklehurst's textbook of geriatric medicine and gerontology eighth edition. Philadelphia: Elsevier 2017, pp. 160-5.
5 Hubbard R, O'Mahoney M, Woodhouse K. Medication prescribing in frail older people. Eur J Clin Pharmacol 2013;69:319-26.

6 Shi S, Klotz U. Age-related changes in pharmacokinetics. Curr Drug Metab 2011;12:601-10.

7 Grandison MK, Boudinot FD. Age-related changes in protein binding of drugs: implications for therapy. Clin Pharmacokinet 2000;38:271-90.

8 Toornvliet R, van Berckel BNM, Luurtsema G, et al. Effect of age on functional $\mathrm{P}$ glycoprotein in the blood-brain barrier measured by use of (R)-[11C]verapamil and positron emission tomography. Clin Pharmacol Ther 2006;79:540-8.

9 McLachlan AJ, Pont LG. Drug metabolism in older peopleA key consideration in achieving optimal outcomes with medicines. J Gerontol A Biol Sci Med Sci 2012;67A:17580.

10 Schwartz JB. The current state of knowledge of age, sex, and their interactions on clinical pharmacology. Clin Pharmacol Ther 2007;82:87-96.

11 Cockroft DW, Gault MH. Prediction of creatinine clearance from serum creatinine. Nephron 1976;16:31-41.

12 'Hanlon JT, Aspinall SL, Semla TP, et al. Consensus guidelines for oral dosing of primarily renally cleared medications in older adults. J Am Geriatr Soc 2009;57:335-40.

13 Turner MJ, Mier CM, Spina RJ, et al. Effects of age and gender on the cardiovascular responses to isoproterenol. J Gerontol A Biol Sci Med Sci 1999;54:B393-400.

14 Trifior G, Spina E. Age-related changes in pharmacodynamics: focus on drugs acting on central nervous and cardiovascular systems. Curr Drug Metab 2011;12:611-20.

15 Bowie MW, Slattum PW. Pharmacodynamics in older adults: a review. Am J Geriatr Pharmacother 2007;5:263303.

16 Thompson CM, Johns DO, Sonawane B, et al. Database for physiologically based pharmacokinetic (PBPK) modeling: physiological data for healthy and health-impaired elderly. J Toxicol Environ Health B Crit Rev 2009;12:1-24.

17 Sleeper R. Common geriatric syndromes and special problems. Consult Pharm 2009;24:447-62.

18 Seymour RM, Routledge PA. Important drug-drug interactions in the elderly. Drugs Aging 1998;12:485-94.

19 Mason P. Important drug-nutrient interactions. Proc Nutr Soc 2010;69:551-7.

20 Moore AA, Whiteman EJ, Ward KT. Risks of combined alcohol/medication use in older adults. Am J Geriatr Pharmacother 2007;5:64-74.

21 Hansten PD, Horn JR, Koda-Kimble MA, et al. Drug interactions: a clinical perspective and analysis of current developments. Vancouver: Applied Therapeutics 2000.

22 Skalli S, Zaid A, Soulaymani R. Drug interactions with herbal medicines. Ther Drug Monit 2007;29:679-86.

23 Spinewine A, Schmader KE, Barber N, et al. Appropriate prescribing in elderly people: how well can it be measured and optimised? Lancet 2007;370:173-84.

24 Gallagher P, Ryan C, Byrne S, et al. STOPP (Screening Tool of Older Person's Prescriptions) and START (Screening 
Tool to Alert doctors to Right Treatment). Consensus validation. Int J Clin Pharmacol Ther 2008;46:72-83.

25 Siripala UGS, Premadasa SPK, Samaranayake NR, et al. Usefulness of STOPP/START criteria to assess appropriateness of medicines prescribed to older adults in a resource - limited setting. Int J Clin Pharm 2019;41:525-30. https://doi.org/10.1007/s11096-019-00786-7

26 Kara O, Arik G, Kizilarslanoglu MC, et al. Potentially inappropriate prescribing according to the STOPP/START criteria for older adults. Aging Clin Exp Res 2016;28:761-8. https://doi.org/10.1007/s40520-015-0475-4

27 Williams S, Miller G, Khoury R, et al. Rational deprescribing in the elderly. Ann Clin Psychiatry 2019;31:144-52.

28 Eibling D. Frailty and polypharmacy in older patients with otolaryngologic diseases. Clin Geriatr Med 2018;34:28998. https://doi.org/10.1016/j.cger.2018.01.006

29 Panel on Prevention of Falls in Older Persons, American Geriatrics Society and British Geriatrics Society. Summary of the updated American Geriatrics Society/British Geriatrics Society clinical practice guideline for prevention of falls in older persons. J Am Geriatr Soc 2011;59:148-57. https://doi.org/10.1111/j.1532-5415.2010.03234.x

30 DelGaudio JM, Panella NJ. Presbynasalis. Int Forum Allergy Rhinol 2016;6(:1083-7. https://doi.org/10.1002/alr.21787

31 Rodriguez K, Rubinstein E, Ferguson BJ. Clear anterior rhinorrhea in the population. Int Forum Allergy Rhinol 2015;5:1063-7. https://doi.org/10.1002/alr.21583

32 Hotta H, Uchida S. Aging of the autonomic nervous system and possible improvements in autonomic activity using somatic afferent stimulation. Geriatr Gerontol Int 2010;10(Suppl 1):S127-36. https://doi.org/10.1111/ j.1447-0594.2010.00592.x

33 Mirza N, Kroger H, Doty RL. Influence of age on the 'nasal cycle'. Laryngoscope 1997;107:62-6. https://doi. org/10.1097/00005537-199701000-00014

34 Eibling D. Too many medications - not enough saliva. Ear Nose Throat J 2019;98:263-4. https://doi.org/ 10.1177/0145561319839704

35 Miranda-Rius J, Brunet-Llobet L, Lahor-Soler E, et al. Salivary secretory disorders, inducing drugs, and clinical management. Int J Med Sci 2015;12:811-82. https://doi. org/10.7150/ijms.12912

36 Villa A, Wolff A, Aframian D, et al. World Workshop on Oral Medicine VI: a systematic review of medication-induced salivary gland dysfunction: prevalence, diagnosis, and treatment. Clin Oral Invest 2015;19:1563-80. https://doi. org/10.1007/s00784-015-1488-2

37 Villa A, Wolff A, Narayana N, et al. World Workshop on Oral Medicine VI: a systematic review of medication-induced salivary gland dysfunction. Oral Diseases 2016;22:365-82. https://doi.org/10.1111/odi.12402

38 Wolff A, Joshi RK, Ekstrom J, et al. A guide to medications inducing salivary gland dysfunction, xerostomia, and subjective sialorrhea: a systematic review sponsored by the World Workshop on Oral Medicine VI. Drugs R D 2017;17:1-28 https://doi.org/10.1007/s40268-016-0153-9 